Supporting Information for

\title{
Supramolecular Depolymerization of Nanowires Self-Assembled from Micelles
}

Hongbing Gao ${ }^{\dagger}$, Liang Gao ${ }^{\dagger}$, Jiaping Lin*, Yingqing Lu, Liquan Wang, Chunhua Cai* and Xiaohui Tian

Shanghai Key Laboratory of Advanced Polymeric Materials, Key Laboratory for Ultrafine Materials of Ministry of Education, School of Materials Science and Engineering, East China University of Science and Technology, Shanghai 200237, China

$\dagger$ These authors contributed equally to this work.

*Corresponding author. E-mail: jlin@ecust.edu.cn, caichunhua@ecust.edu.cn 


\section{Contents}

1. Polymer synthesis and micelle solution preparation S1

2. Aggregate structure characterizations S5

3. Definitions and measurements of the parameters $X_{\mathrm{n}}, f_{\mathrm{X}}$, and $Ð$ S7

4. Theoretical model proposed for kinetics of supramolecular depolymerization S8

5. Stability studies of the pre-assembled subunits S11

6. Re-polymerization of the subunits depolymerized from the nanowires $\mathrm{S} 12$

7. Variation in the $f_{X}$ of the nanowires with depolymerization time $\mathrm{S} 13$

8. Estimation of the concentration of aggregates by static light scattering (SLS) S 14

9. Variation in the $R_{\mathrm{h}}$ of the nanowires at different temperatures S17

10. Effect of the inter-micelle interaction on the depolymerization S18 


\section{Polymer synthesis and micelle solution preparation}

1.1. Reagents and Materials. Polyethylene glycol monomethyl ether (mPEG-OH, $\left.M_{\mathrm{w}}=750\right)$ were purchased from Sigma Inc. Analytical grade of ethyl acetate, tetrahydrofuran (THF), and 1,4-dioxane were refluxed with sodium and distilled immediately before use. All the other reagents were bought from Adamas-beta and purified according to conventional methods or used as received. Deionized water was prepared in a Millipore Super-Q Plus Water System to a level of $18.2 \mathrm{M} \Omega \mathrm{cm}$ resistance. The dialysis bag (Membra-cel, 3500 molecular weight cutoff) was purchased from Serva Electrophoresis $\mathrm{GmbH}$.

1.2 Synthesis of PBLG homopolymer. $\gamma$-benzyl-L-glutamate- $N$-carboxyanhydride (BLG-NCA) was synthesized according to the procedure reported in the literatures. ${ }^{[\mathrm{S} 1-\mathrm{S} 3]}$ PBLG homopolymer was synthesized by ring-opening polymerization of BLG-NCA initiated by triethylamine with 1 , 4-dioxane as the solvent. The reaction was performed for 3 days, at the end of the polymerization, the viscous reaction mixture was poured into a large volume of anhydrous ethanol. The precipitated product was dried under vacuum and then purified twice by repeated precipitation from a chloroform solution into a large volume of anhydrous methanol. The molecular weight of the PBLG homopolymers was obtained from the gel permeation chromatography (GPC) analysis (PL-GPC 50, DMF as eluent solvent, $50^{\circ} \mathrm{C}$ ). The calibration curve was obtained by using narrow polydispersity PBLG homopolymer as the standards $\left(\bigoplus_{\mathrm{PBLG}}=1.12 \sim 1.15\right)$. The weight average molecular weight $\left(M_{w}\right)$ of the PBLG homopolymer was estimated to be 120000 with a $\bigoplus_{\text {polymer }}$ of 1.26 (Figure S1). 


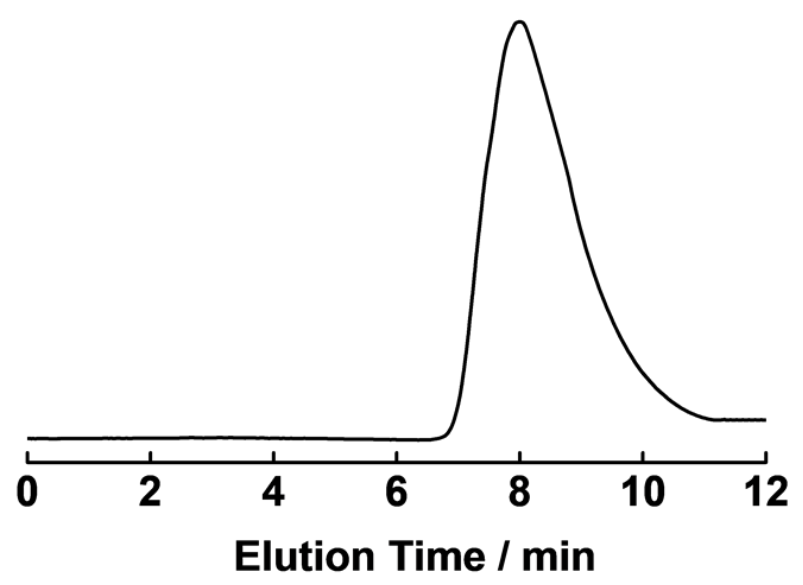

Figure S1. GPC trace of PBLG homopolymer using DMF as eluent.

1.3 Synthesis of the PBLG-g-PEG copolymer. PBLG-g-PEG graft copolymers were prepared by ester exchange reaction of PBLG homopolymer with mPEG-OH. ${ }^{[\mathrm{S} 3-\mathrm{S} 5]}$ Feeding ratio of PBLG homopolymer to mPEG-OH was 1 (i.e. $\mathrm{n}_{\mathrm{BLG}} / \mathrm{n}_{\mathrm{PEG}}, \mathrm{mol} / \mathrm{mol}, 1 / 1$ ). The reaction was performed at $55{ }^{\circ} \mathrm{C}$ in 1,2 -dichloroethane with $p$-toluenesulfonic acid as a catalyst, ${ }^{[\mathrm{S} 6]}$ and the reaction time is approximately $1 \mathrm{~h}$. The reaction mixture was then precipitated in a large volume of anhydrous methanol. The product was purified twice by repeatedly precipitating from a chloroform solution into a large volume of anhydrous methanol and dried under vacuum. The composition of the graft copolymer was determined by ${ }^{1} \mathrm{H}$ NMR spectrum (Avance 550, Bruker) using deuterated chloroform $\left(\mathrm{CDCl}_{3}\right)$ with $15 \mathrm{vol} \%$ deuterated trifluoroacetic acid (TFA-d) as the solvent and tetramethylsilane (TMS) as the internal standard. The degree of grafting is defined as the ratio of the number of PEG chains to the degree of polymerization of the polypeptide backbone, which can be calculated from the intensity ratio of the ethylene proton signal $(\delta=3.6 \mathrm{ppm})$ of PEG to that of the methylene proton signal $(\delta=5.1 \mathrm{ppm})$ of PBLG in the ${ }^{1} \mathrm{H}$ NMR spectrum (Figure S2). According to the NMR analysis, the degree of grafting was determined to be $2.33 \%$. The weight percentage of PEG is $7.55 \mathrm{wt} \%$. 


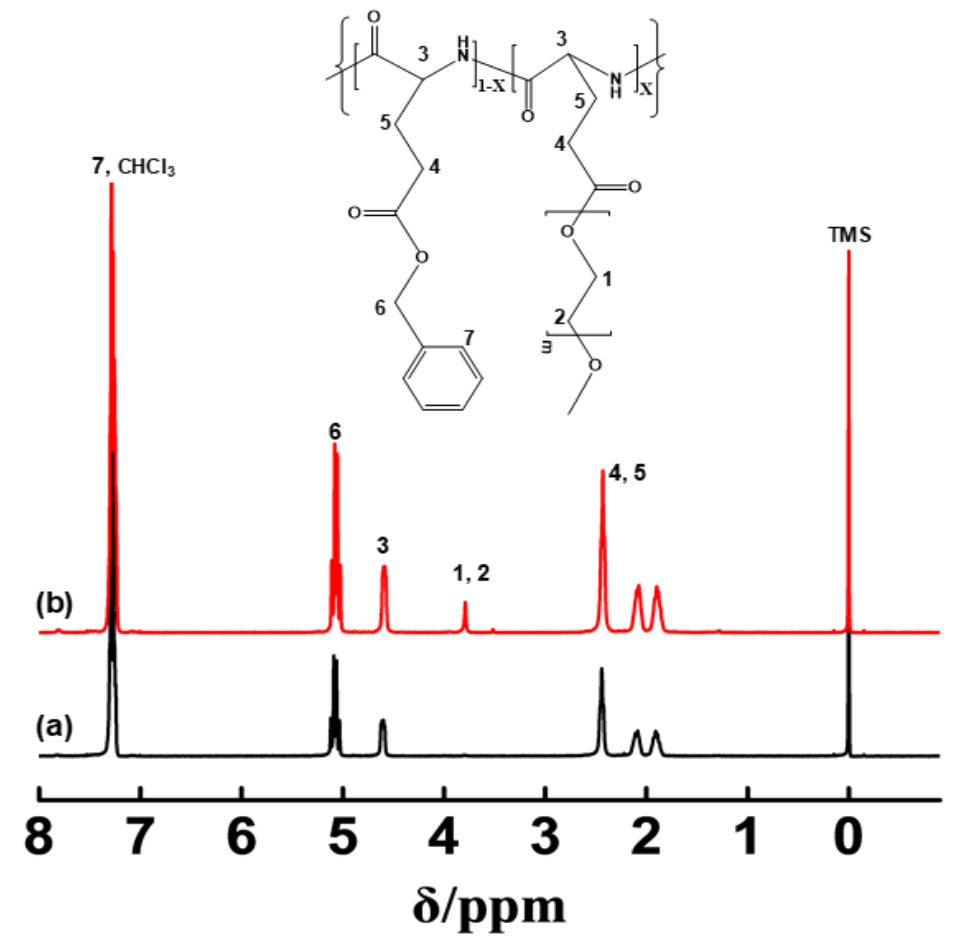

Figure S2. ${ }^{1} \mathrm{H}$ NMR spectra of (a) PBLG homopolymer and (b) PBLG-g-PEG graft copolymer in $\mathrm{CDCl}_{3}$.

1.4 Micelle solution preparation. The subunit aggregates were prepared by a selective precipitation method. First, the graft copolymers of PBLG- $g$-PEG were dissolved in THF/DMF $(1 / 1, \mathrm{v} / \mathrm{v})$ mixture solvents to obtain stock solutions. The polymer concentration of the stock solution is $0.4 \mathrm{~g} / \mathrm{L}$. Then $1.5 \mathrm{~mL}$ of deionized water was added to $10 \mathrm{~mL}$ of the PBLG- $g$-PEG initial solution with vigorous stirring to form subunits. The experimental process to prepare subunits was conducted at the constant temperature of $40{ }^{\circ} \mathrm{C}$. After stabilized for at least $10 \mathrm{~h}$, the solution was subjected to $5{ }^{\circ} \mathrm{C}$ and annealed at this temperature. One dimensional (1D) nanowires with nodes were formed through supramolecular polymerization of the subunits in an end-to-end manner.

To investigate the degradation of nanowires in solution, the nanowire solution was heated to and annealed respectively at $40{ }^{\circ} \mathrm{C}, 35^{\circ} \mathrm{C}, 33{ }^{\circ} \mathrm{C}, 30^{\circ} \mathrm{C}$, and $20^{\circ} \mathrm{C}$. To observe the temporal variation of the micelle morphologies at each temperature, we pipetted $0.5 \mathrm{~mL}$ of sample solution into a large amount of water $(c a .15 \mathrm{~mL})$ to freeze the morphologies at various time intervals. The sample 
solution was dialyzed against deionized water for 3 days to ensure that all the organic solvents were removed. It should be noted that after the formation of aggregates, the storage time has a negligible effect on the morphology. For example, morphological changes were not observed when the micelles were aged for more than 3 months. 


\section{Aggregate structure characterizations}

2.1 Transmission Electron Microscopy (TEM). The morphologies of aggregates were examined by TEM (JEM-1400, JEOL) operated at an accelerating voltage of $100 \mathrm{kV}$. The sample was prepared by placing drops of solution on a copper grid coated with carbon film and then were dried at room temperature.

2.2 Scanning Electron Microscopy (SEM). The morphologies of the aggregates were also observed by SEM (S4800, HITACHI) operated at an accelerating voltage of $15 \mathrm{kV}$. The samples were prepared by placing a drop of solution on a copper grid coated with carbon film and then were dried at room temperature. Before the observations, the samples were sputtered by Platinum.

2.3 Dynamic (DLS) and Static Light Scattering (SLS) Measurements. DLS measurements were performed at a scattering angle of $90^{\circ}$ on a commercial LLS spectrometer (ALV/CGS-5022) equipped with an ALV-High QE APD detector and an ALV-5000 digital correlator using a He-Ne laser (the wavelength $\lambda=632.8 \mathrm{~nm}$ ) as light source. For the DLS measurements, the intensity correlation function was measured at the same temperatures with that of the sample annealing. The obtained intensity autocorrelation function, $G^{(2)}(t)$, is related to the electric field autocorrelation function, $g^{(1)}(t)$, by means of the Siegert relation. $g^{(1)}(t)$ is further related to the characteristic linewidth $(\Gamma)$ distribution $G(\Gamma)$. Here, $G(\Gamma)$ can be calculated by a Laplace inversion of $g^{(1)}(t)$ using the CONTIN program. From the expression $\Gamma=D_{\text {app }} q^{2}$, the apparent translational diffusion coefficients $\left(D_{\text {app }}\right)$ were determined. $\Gamma$ is the decay rate, which is the inverse of the relaxation time $(t)$. $q$ is the scattering vector defined as $q=(4 \pi n \sin (\theta / 2) / \lambda)$ (where $n$ is the refractive index of the solution, $\theta$ is the scattering angle, and $\lambda$ is the wavelength of the incident laser light in vacuum). The apparent hydrodynamic radius $\left(R_{\mathrm{h}}\right)$ can be determined by the Stokes-Einstein relationship $R_{\mathrm{h}}=$ $k_{\mathrm{B}} T /\left(6 \pi \eta D_{\mathrm{app}}\right)$, where $k_{\mathrm{B}}, T$, and $\eta$ are the Boltzmann constant, the absolute temperature, and the solvent viscosity, respectively. 
SLS measurements were performed to estimate the average aggregation number of polymers in one subunit $\left(n_{s u b}\right)$. In order to exclude the influence of temperature on the density of the micelles in solution, before the SLS measurements, the micelle solution was first added into a large amount of water to freeze the structures and subsequently dialyzed in water for 3 days to ensure all the organic solvents were removed. When the micelles were frozen, the density of the aggregates exhibited few changes in a small temperature range. ${ }^{[\mathrm{S} 7, \mathrm{~S} 8]}$ During the SLS measurements, we ensured that the subunit solutions were in the same environment. The $d n / d c$ value of PBLG homopolymers and PEG in water has been reported in the literatures, ${ }^{[\mathrm{S} 9-\mathrm{S} 11]}$ which are $0.131 \mathrm{~mL} / \mathrm{g}$ and $0.133 \mathrm{~mL} / \mathrm{g}$, respectively. The value of $0.131 \mathrm{~mL} / \mathrm{g}$ is adopted for the graft copolymers in the present work, since the weight fraction of PEG chains is low $(\sim 0.07)$ for the PBLG- $g$-PEG graft copolymers. 


\section{Definitions and measurements of the parameters $X_{\mathrm{n}}, f_{X}$, and $\boldsymbol{D}$}

\subsection{Number-average degree of polymerization of the nanowires $\left(X_{\mathrm{n}}\right)$}

The parameter $X_{\mathrm{n}}$ characterizes the number-average degree of polymerization of the nanowires and is given by

$$
X_{\mathrm{n}}=\frac{\sum_{X} X n_{X}}{\sum_{X} n_{X}}
$$

where $n_{X}$ is the number of nanowires with the degree of polymerization $X$. In the experiments, the parameter $n_{X}$ was obtained by collecting TEM images with more than 400 aggregates (the images were analyzed using the Image-Pro Plus software).

\subsection{Number fraction $f_{X}$ of the nanowire with degree of polymerization $X$}

The parameter $f_{X}$ characterizes the number fraction of the nanowires with the degree of polymerization $X$ in the systems. The number fraction is given as

$$
f_{\mathrm{X}}=\frac{n_{\mathrm{X}}}{\sum_{\mathrm{X}} n_{\mathrm{X}}}
$$

\subsection{Polydispersity index $(\bigoplus)$}

The parameter $Ð$ characterizes the size distribution of the nanowires and is given by

$$
Ð=\frac{\sum_{\mathrm{X}} \mathrm{X}^{2} n_{\mathrm{x}} \sum_{\mathrm{X}} n_{\mathrm{X}}}{\left(\sum_{\mathrm{X}} \mathrm{X} n_{\mathrm{X}}\right)^{2}}
$$




\section{Theoretical model proposed for kinetics of supramolecular depolymerization}

Herein, we developed a theoretical model to describe the evolution from the nanowires to the micellar subunits in the experiments, which was inspired by the classical degradation theory of polymer chains. ${ }^{[\mathrm{S} 12-\mathrm{S} 15]}$ In this depolymerization process, the degradation of the nanowires and chain transfer between two neighboring subunits on the nanowire simultaneously occur. In the present work, the polymer chain transfer refers to the transfer of the polymer chains between the neighboring subunits on the nanowires. The PBLG-g-PEG graft copolymers can self-assemble into spindle-like micelles. Both experiments and theoretical simulations showed that the rigid PBLG backbones align and are oriented along the long axis in the micelle. This orientation leads to the heterogeneous coverage of PEG around the cores and some exposure of the core to solvent at the micelle ends. ${ }^{[\mathrm{S} 16]}$ At lower temperatures, the PBLG tends to be more hydrophobic; consequently, the micelle ends become reactive and induce polymerization. When the temperature is increased, the physical interaction between the connected micelle subunits is disrupted, and the nanowire is randomly depolymerized into fragments and subunits. Meanwhile, in the higher-temperature depolymerization, the hydrophobicity of copolymers decreases and molecular motion increases. Chain transfer between the neighboring micelles therefore occurs since the micelle ends are partially opened.

We suppose the degradation of the nanowires contains two processes: the degradation and the chain transfer. In the degradation, the nanowires randomly degrade into several parts. Due to chain transfer in the process of depolymerization, subunits with various size were obtained. We considered chain transfer mainly occurs between two neighboring subunits. In an extreme case, the chain transfer is sufficient and all chains in one subunit transfer into the neighboring subunit, which gives rives to the formation of one larger subunit (i.e., the occurrence of subunit fusion). (Scheme S1). 


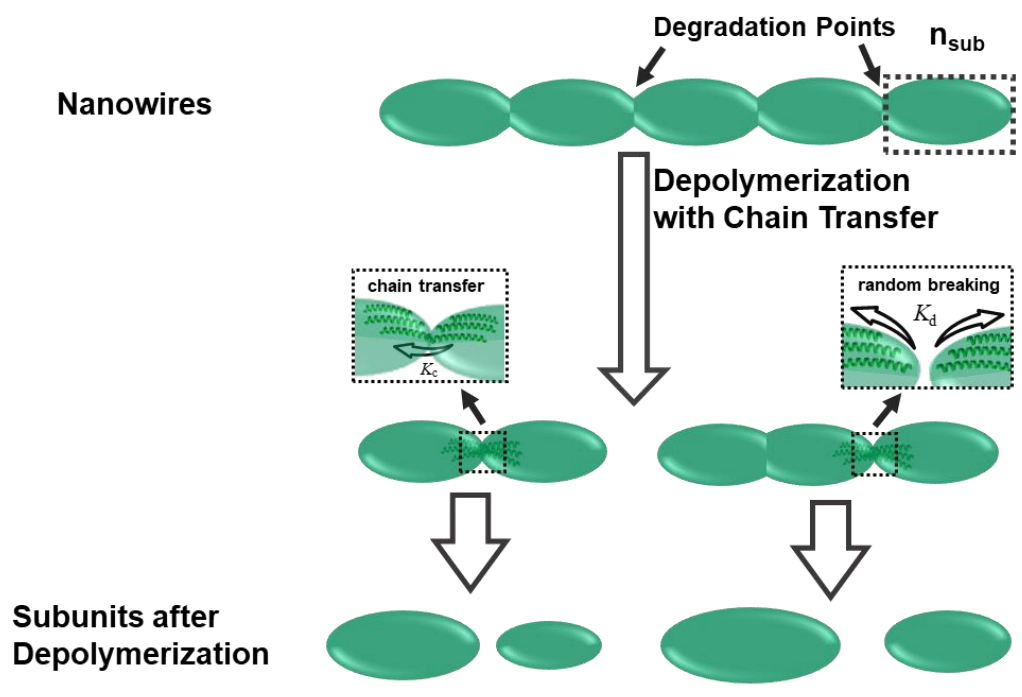

Scheme S1. Schematic of the supramolecular degradation process of the nanowires in solution.

Due to the material conservation of the micelle system, the total number of copolymer chains in the solution, $N_{\text {total }}$, can be expressed as

$N_{\text {total }}=N_{\text {sub }} \cdot n_{\text {sub }}=X_{n} \cdot N \cdot n_{\text {sub }}$

where $N_{\text {sub }}$ and $n_{\text {sub }}$ are number of subunits in the solution and number average aggregation number of polymer chains per subunit, respectively. We suppose that the subunits before polymerization is monodisperse. $X_{\mathrm{n}}$ and $N$ are number-average degree of polymerization of the nanowires (the number average number of subunits per nanowire) and total number of nanowires in the solution, respectively.

For any nanowire micelles undergoing degradation, the following relationship remains valid

$n_{\text {sub }} \cdot X_{n} \cdot N=N_{\text {total }}$

Then, the differential equation of Eq. (S-5) is written as

$n_{\text {sub }} \cdot X_{n} \cdot \frac{d N}{d t}+n_{\text {sub }} \cdot N \cdot \frac{d X_{n}}{d t}+N \cdot X_{n} \cdot \frac{d n_{s u b}}{d t}=0$

We assumed that the process of nanowire depolymerization follows the classical kinetics theory of random depolymerization. During the depolymerization of nanowires, the population of micelles depolymerized from nanowires increases with increasing degradation time. The number variation of the micelles with depolymerization time was given by Eq. (S-7). Additionally, the chain transfer 
between two neighboring subunits is considered as a second-order reaction model; that is, the variation of $n_{s u b}$ in the subunit depends on the rate constant of chain transfer and the aggregation number of polymer chains of two neighboring subunits. Therefore, the variation in $n_{\text {sub }}$ with depolymerization time was given by Eq. (S-8). The kinetic equations can be written as

$\frac{d N}{d t}=K_{d} \cdot N$

$\frac{d n_{s u b}}{d t}=K_{c} \cdot n_{s u b}^{2}$

where $K_{d}$ is the apparent rate constant for degradation at random. And $K_{c}$ is the rate constant for chain transfer between the micelles. Substituting Eq. (S-7) and (S-8) in (S-6) yields,

$X_{n} \cdot K_{d}+\frac{d X_{n}}{d t}+X_{n} \cdot K_{c} \cdot n_{s u b}=0$

Integrating (S-9) then affords an expression for the $X_{\mathrm{n}}$ of the nanowires as a function of time, and $X_{0}$ in terms of the initial number-average degree of polymerization

$\ln \frac{X_{n}}{X_{0}}=-\left(K_{d} t+K_{c} \int_{0}^{t} n_{s u b} d t\right)$

According to the classical mean value theorem of integrals, the integration of $\int_{0}^{t} n_{s u b} d t$ can be approximated as $\bar{n}_{s u b} t$, where $\bar{n}_{s u b}$ is the average value of $n_{s u b}$ in the depolymerization process. Therefore, the final expression for $X_{\mathrm{n}}$ of the nanowires versus depolymerization time $t$ can be rewritten as

$X_{n}=X_{0} e^{-\left(K_{d}+K_{c} \cdot \bar{n}_{s u b}\right) t}$

by defining the total rate constant $K=K_{d}+K_{c} \cdot \bar{n}_{s u b}$, Eq. (S-11) can be simplified as

$X_{n}=X_{0} e^{-K t}$

Subsequently, we used Eq. (S-12) to fit the experimental data of $X_{\mathrm{n}}$ as a function of time. It was found that the experimental data can be fitted with the equation (the lines in Figure $3 \mathrm{a}$ and Figure $5 \mathrm{~g}$ in the main text). The rate constant $K$ can be obtained from the fitting results. 


\section{Stability studies of the pre-assembled subunits}

In the experiment, the spindle-like subunits were first prepared in DMF/THF/water solution at $40{ }^{\circ} \mathrm{C}$; this solution was then stored at $40^{\circ} \mathrm{C}$. The variation in size of these subunits as a function of stabilization time was examined. As shown in Figure S3a-c, no substantial variation in morphology of the spindle-like subunits is observed after stabilization for 30 days at $40{ }^{\circ} \mathrm{C}$. Statistics reveal that the average length and diameter are nearly unchanged over time (Figure S3d, the data were obtained by collecting TEM images with more than 200 subunits). In addition, we examined the variation in hydrodynamic radius $\left(R_{\mathrm{h}}\right)$ of the subunits with time at $40{ }^{\circ} \mathrm{C}$ by DLS. As shown in Figure S3e, the $R_{\mathrm{h}}$ value and distribution of $R_{\mathrm{h}}$ remain almost unchanged, which indicates that the subunits in solution at $40{ }^{\circ} \mathrm{C}$ are stable over time. These results demonstrate that the individual subunits in solution are stable and that the polymer chain transfer only occurs between the neighboring subunits on the nanowires in the depolymerization.

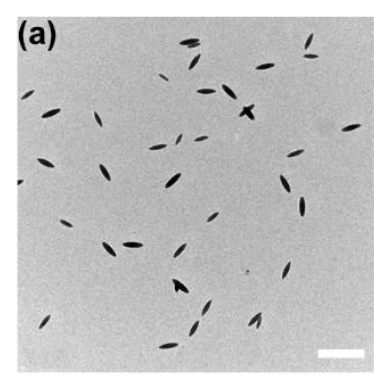

(d)

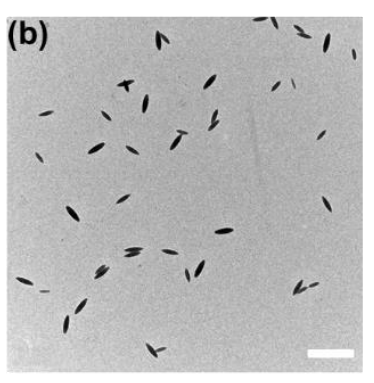

(e)

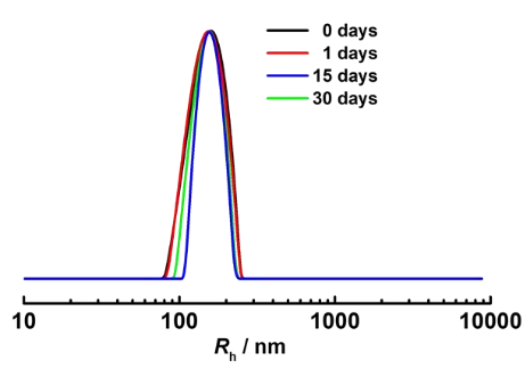

Figure S3. (a-c) TEM images of pre-assembled subunits formed by PBLG- $g$-PEG graft copolymers at $40{ }^{\circ} \mathrm{C}$ with various stabilizing times: (a) 0 days; (b) 15 days; and (c) 30 days. Scale bars: $1 \mu \mathrm{m}$. (d) Statistics for variation in the average length and diameter of the subunits with time. (e) DLS testing for hydrodynamic radius $\left(R_{\mathrm{h}}\right)$ of the subunits in solution at $40{ }^{\circ} \mathrm{C}$ with various stabilizing times. The scattering angle is $90^{\circ}$. 


\section{Re-polymerization of the subunits depolymerized from the nanowires}

Figure S4 shows the reversible phenomenon of the supramolecular polymerization and depolymerization. As shown in the figure, when the subunit solution was annealed at a lower temperature $\left(5^{\circ} \mathrm{C}\right)$, the nanowires were obtained through the supramolecular polymerization of subunits. With increasing temperature to $40{ }^{\circ} \mathrm{C}$, the nanowires depolymerize into subunits. The subunits degraded from the nanowires become reactive and can polymerize into nanowires again when changing the temperature from $40{ }^{\circ} \mathrm{C}$ to $5{ }^{\circ} \mathrm{C}$. These results demonstrate that the supramolecular polymerization of the subunits and depolymerization of nanowires are reversible.

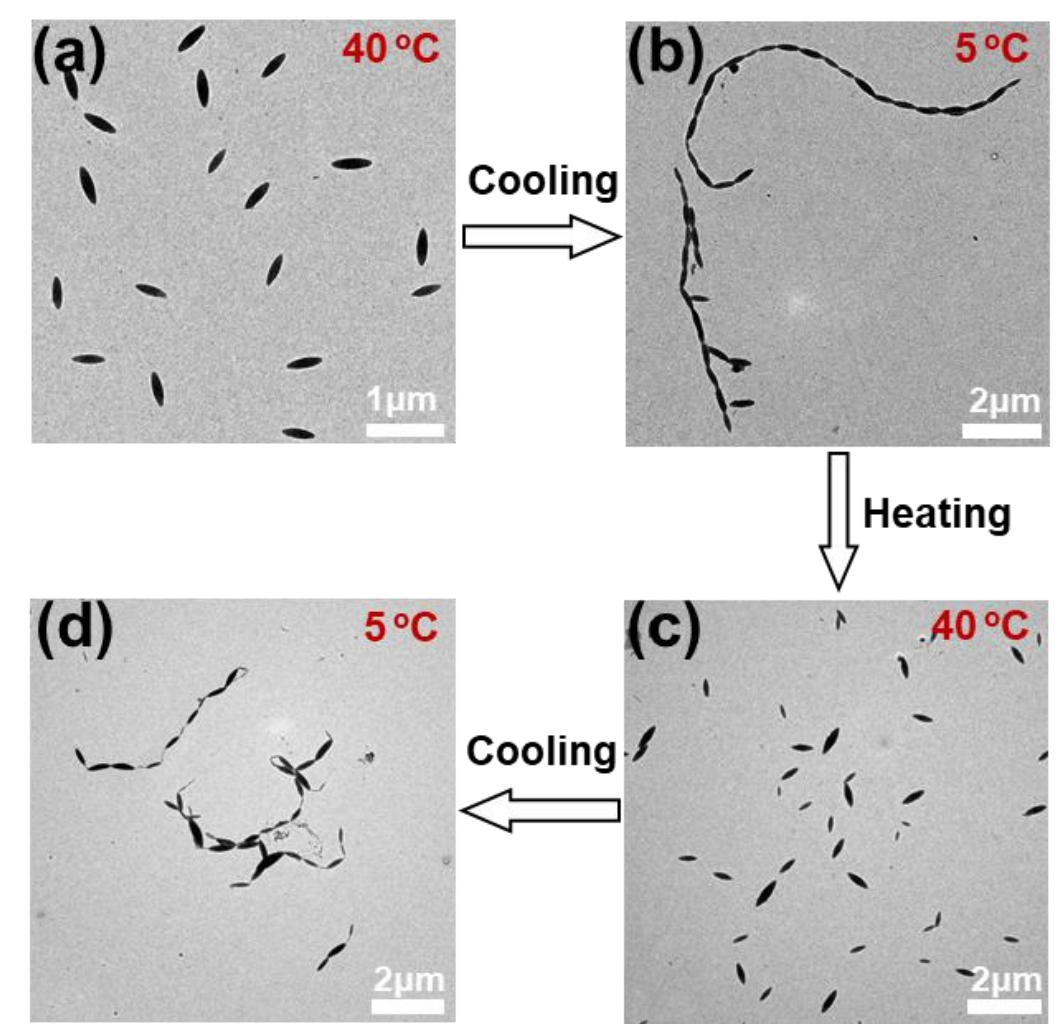

Figure S4. (a) TEM image of the initial subunits self-assembled from PBLG-g-PEG graft copolymers at $40{ }^{\circ} \mathrm{C}$; (b) TEM image of the nanowires formed through the supramolecular polymerization of the initial subunits at $5{ }^{\circ} \mathrm{C}$; (c) TEM image of the micellar subunits depolymerized from the nanowires at $40{ }^{\circ} \mathrm{C}$; (d) TEM image of the nanowires polymerized from the subunits again at $5{ }^{\circ} \mathrm{C}$, and these subunits were obtained by depolymerizing of the nanowires at $40{ }^{\circ} \mathrm{C}$. 


\section{Variation in the $f_{X}$ of the nanowires with depolymerization time}

The number fraction, $f_{\mathrm{x}}$, of the depolymerized nanowires with the degree of polymerization $X$ at various depolymerization time $t$ was examined. The nanowire solution was annealed at $40{ }^{\circ} \mathrm{C}$. The statistical results were shown in Figure S5a. As can be seen, the number fraction of the subunits $(X=$ 1) increases with time and reaches to 1 finally after depolymerization of $48 \mathrm{~h}$. Note that the value of $f_{X}$ has no changes with time after $48 \mathrm{~h}$, which indicates that the depolymerization of the nanowires has reached equilibrium. Figure S5b-c shows TEM images of the subunits depolymerized from nanowires for $48 \mathrm{~h}$ and $120 \mathrm{~h}$, respectively. As can be seen, the morphologies of the subunits depolymerized from the nanowires show no obvious changes after $48 \mathrm{~h}$.
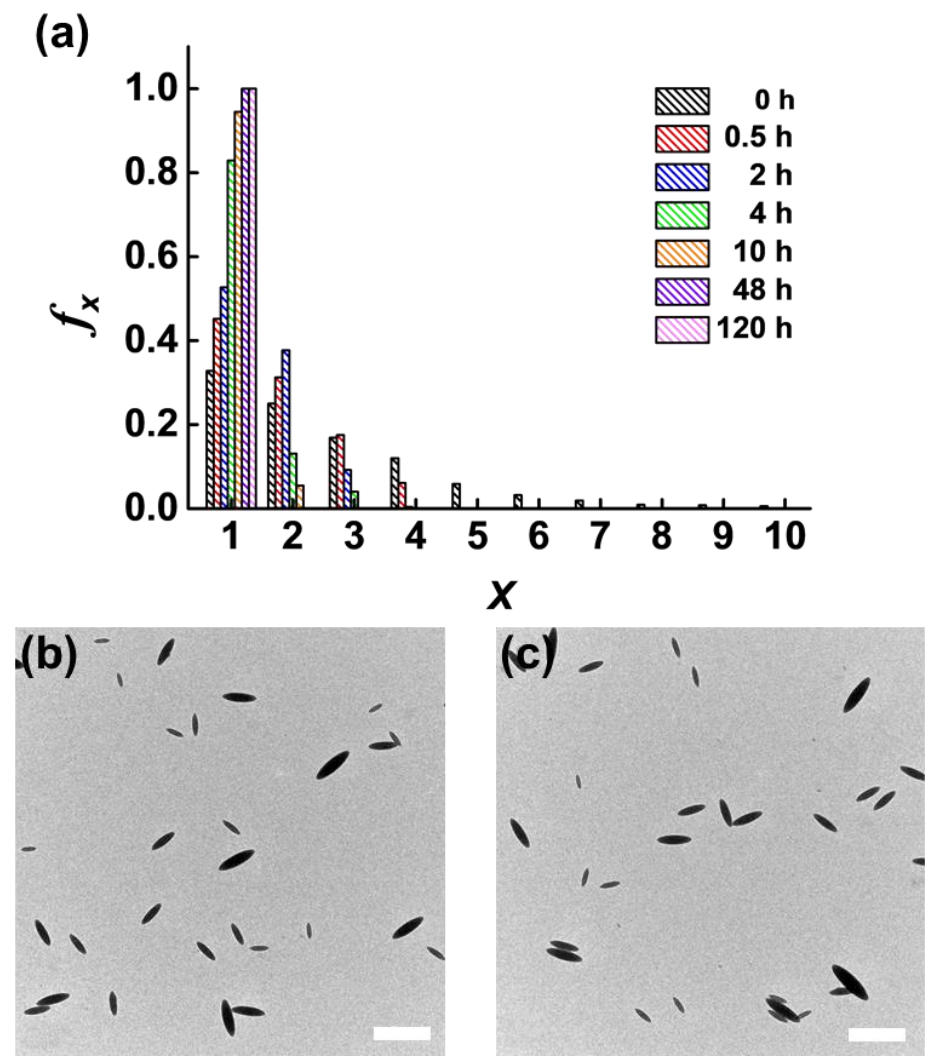

Figure S5. (a) Number fraction $\left(f_{X}\right)$ of the nanowires as a function of $X$ at various depolymerization time $t$ at $40^{\circ} \mathrm{C}$. (b) and (c) TEM images of the subunits depolymerized from nanowires for $48 \mathrm{~h}$ and $120 \mathrm{~h}$, respectively. Scale bars: $1 \mu \mathrm{m}$. 


\section{Estimation of the concentration of aggregates by static light scattering (SLS)}

To obtain the concentration of aggregates, the average aggregation number of polymers in one subunit $\left(n_{s u b}\right)$ was estimated by static light scattering (SLS) measurements. In SLS, the angular dependence of the excess absolute time-average scattered intensity of the dilute micelle solutions was measured. We plotted the SLS data with the form factor of an ellipsoid in the SLS characterization. The SLS measurement was performed at scattering angles from $30^{\circ}$ to $150^{\circ}$ (in increments of $2^{\circ}$ ) at $25^{\circ} \mathrm{C}$. The light scattering data were treated with

$K c /_{R}=\frac{1}{M P(q)}+2 A_{2} c+\cdots$

where $K, c, R, M, P(q)$ and $A_{2}$ are the optical constant, the sample concentration, the excess Rayleigh ratio, the particle molar mass, the form factor of the aggregates and the second virial coefficient, respectively. The term $q$ is the scattering wave vector with magnitude given by $q=\frac{4 \pi n_{0}}{\lambda} \sin (\theta / 2)$, where $n_{0}, \lambda$ and $\theta$ are the refractive index of the solvent, the laser wavelength and the scattering angle, respectively. ${ }^{[\mathrm{S} 17-\mathrm{S} 19]}$

When the concentration $c$ tends to be 0 , the equation (S-13) can be given by

$\left(R /{ }_{K c}\right)_{c \rightarrow 0}=M P(q)$

To characterize the spindles, the model of ellipsoid of revolution was used. ${ }^{[\mathrm{S} 20, \mathrm{~S} 21]}$ The form factor for the ellipsoid of revolution can be expressed as:

$P(q)=\int_{0}^{\frac{\pi}{2}} F^{2}\left[q R^{\prime}(R, \varepsilon, \alpha)\right] \sin \alpha d \alpha$

$R^{\prime}(R, \varepsilon, \alpha)=R\left(\sin ^{2} \alpha+\varepsilon^{2} \cos ^{2} \alpha\right)^{1 / 2}$

$F(u)=\frac{3[\sin (u)-u \cos (u)]}{u^{3}}, u=q R^{\prime}(R, \varepsilon, \alpha)$

where $R, \varepsilon$ and $\alpha$ are the semi axes, the axial ratio of the ellipsoid and the inclination angle of the spherical coordinates in reciprocal space. 
For the initial assembled subunits, the concentration of subunits in the experiments was estimated. Taking the initial subunits of PBLG-g-PEG graft copolymers formed at $40{ }^{\circ} \mathrm{C}$ as an example, in the $10.0 \mathrm{~mL}$ initial solution, the mass concentration of polymer chains was $0.4 \mathrm{~g} / \mathrm{L}$. Therefore, the number of polymer chains in the initial solution can be obtained by

$N_{\text {total }}=0.4 \mathrm{~g} / \mathrm{L} \times 0.01 \mathrm{~L} \div 120.1 \mathrm{~kg} / \mathrm{mol}=3.33 \times 10^{-8} \mathrm{~mol}$

Figure S6 shows the SLS data of the spindle-like subunits and the line is the fitting curve using Equation (S-14) according to the model of ellipsoid of revolution. As shown in Figure S6, the scattering data of the spindles can be described by the model of ellipsoid of revolution. The $R$ and $\varepsilon$ were calculated to be $51 \mathrm{~nm}$ and 5.4, respectively. Therefore, the diameter and length of the subunits obtained in SLS studies were $102 \mathrm{~nm}$ and $550 \mathrm{~nm}$, which agrees with the TEM observations $(\sim 100$ $\mathrm{nm}$ in diameter and $\sim 500 \mathrm{~nm}$ in length).

Then the average aggregation number of PBLG- $g$-PEG chains per subunits can be obtained by

$n_{s u b}=\rho V N_{A} / M_{w}=4 \pi \rho \varepsilon R^{3} N_{A} / 3 M_{w}$

where $\rho, V, N_{A}$, and $M_{w}$ are the density of the aggregate, the volume of the subunits $\left(V=4 \pi \varepsilon R^{3} / 3\right)$, the Avogadro constant and the weight average molecular weight of graft copolymers, respectively. The $\rho$ value is $1.263 \mathrm{~g} / \mathrm{cm}^{3}$, which is calculated according to the weight fractions and the densities of the PBLG and PEG segments (the weight faction: $f_{\mathrm{PBLG}}=0.93, f_{\mathrm{PEG}}=0.07$; the densities: $\rho_{\mathrm{PBLG}}=$ $\left.1.278 \mathrm{~g} / \mathrm{cm}^{3}, \rho_{\text {PEG }}=1.092 \mathrm{~g} / \mathrm{cm}^{3}\right) .{ }^{[\mathrm{S} 22, \mathrm{~S} 23]}$ Therefore, the $n_{\text {sub }}$ of the subunit was calculated to be 1.63 $\times 10^{4}$. The initial number of the subunits in the course of polymerization was

$N_{\text {sub }}=N_{\text {total }} / n_{\text {sub }}=3.33 \times 10^{-8} \mathrm{~mol} \div 1.63 \times 10^{4}=2.04 \times 10^{-12} \mathrm{~mol}$

The volume of the solution ( $\left.V_{\text {solution }}\right)$ was $11.5 \mathrm{~mL}$ for the subunits after the addition of selective solution $\left(\mathrm{H}_{2} \mathrm{O}\right)$. As a result, the molar concentration of the subunits was 
$C=2.04 \times 10^{-12} \mathrm{~mol} \div 0.0115 \mathrm{~L}=1.77 \times 10^{-10} \mathrm{~mol} / \mathrm{L}$

The weight-average molecular weight and concentration of the subunits depolymerized from the nanowires can be obtained in the same way. The value of $n_{s u b}$ and $C$ were $6.42 \times 10^{4}$ and $4.57 \times 10^{-11}$ mol/L, respectively. The detailed results are summarized in Table S1.

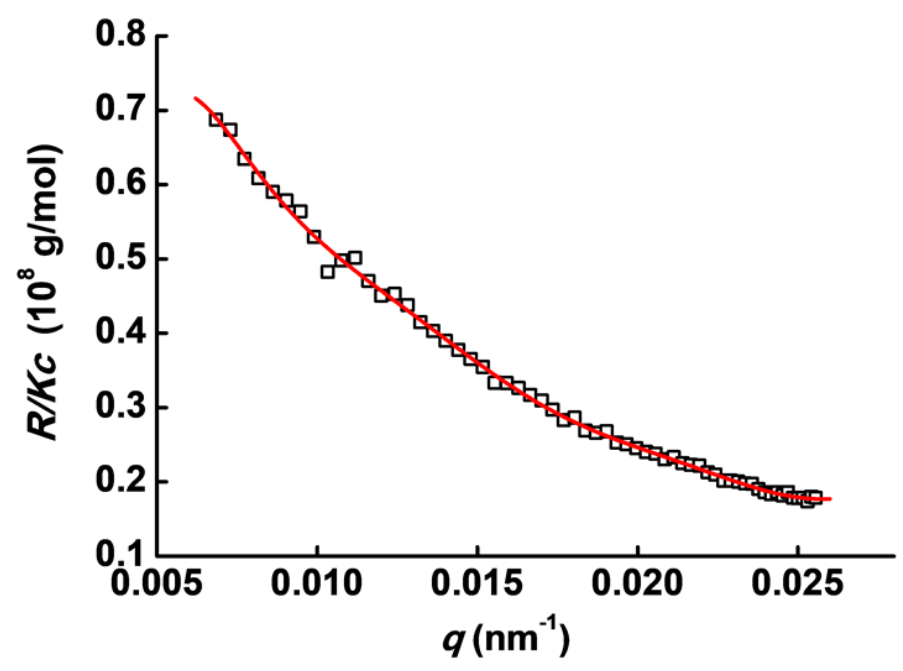

Figure S6. SLS profiles of the spindle-like subunits obtained at $25{ }^{\circ} \mathrm{C}$. The red line is the fitting curve according to the model of ellipsoid of revolution.

Table S1. Laser light scattering characterization of the initial subunits before polymerization and the subunits depolymerized from the nanowires

\begin{tabular}{ccccc}
\hline \multirow{2}{*}{$\begin{array}{c}\text { Temperature } \\
\left({ }^{\circ} \mathrm{C}\right)\end{array}$} & \multicolumn{2}{c}{ Initial subunits } & & Subunits depolymerized from nanowires \\
\cline { 2 - 3 } & $n_{\text {sub }}{ }^{a}$ & $C(\mathrm{~mol} / \mathrm{L})^{b}$ & $n_{\text {sub }}$ & $C(\mathrm{~mol} / \mathrm{L})$ \\
\hline 40 & $1.63 \times 10^{4}$ & $1.77 \times 10^{-10}$ & & $6.42 \times 10^{4}$ \\
\hline
\end{tabular}

${ }^{a}$ The average aggregation number of polymers in one subunit $\left(n_{s u b}\right)$ was obtained by static light scattering (SLS) measurements.

${ }^{b}$ Molar concentration of subunits 


\section{Variation in the $\boldsymbol{R}_{\mathrm{h}}$ of the nanowires at different temperatures}

We also used the DLS to examine the depolymerization of the nanowires in situ. Figure S7 shows the variation in the $R_{\mathrm{h}}$ for the nanowire solution at various temperatures. As shown in the figure, the $R_{\mathrm{h}}$ decreases with time and the value of $R_{\mathrm{h}}$ eventually levels off at a level. In addition, the variation rate in $R_{\mathrm{h}}$ increases with temperature, which is consistent with the statistics results of the electron microscope observations (main text, Figure 4a).

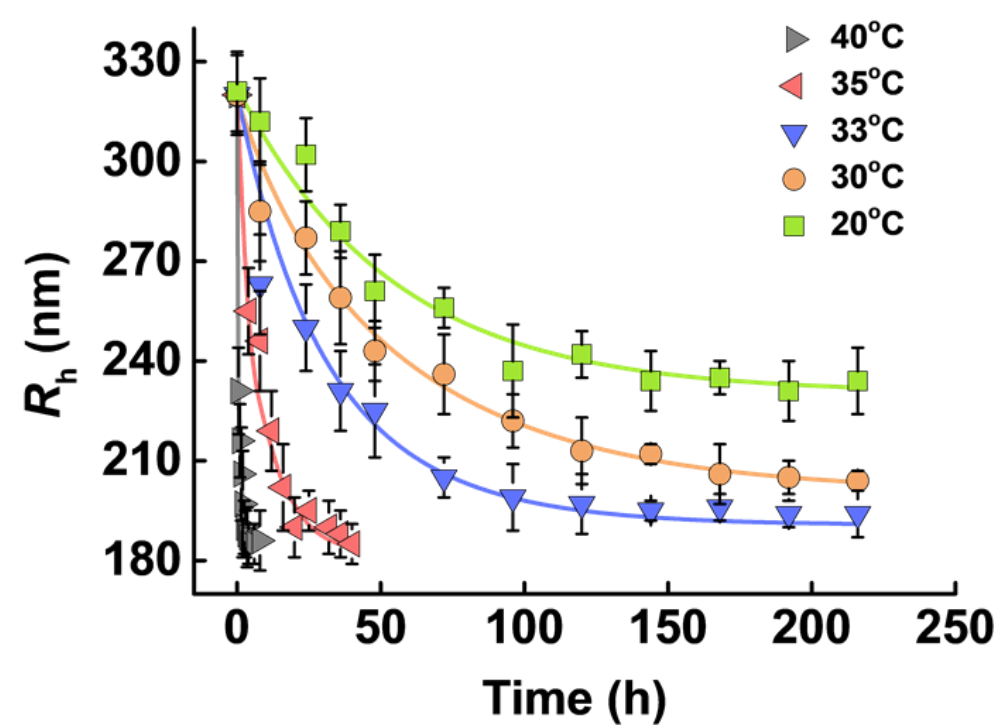

Figure S7. Plots of the hydrodynamic radius $\left(R_{\mathrm{h}}\right)$ as a function of time at different temperatures. The $R_{\mathrm{h}}$ was measured at the scattering angle of $90^{\circ}$. 


\section{Effect of the inter-micelle interaction on the depolymerization}

\subsection{Effect of the inter-micelle interaction on the morphology changes of the depolymerization}

The water content has an important effect on the strength of the non-covalent bond interaction, which is caused by the hydrophobicity of PBLG blocks. The strength of the physical interaction increases with the content of added water. Therefore, we examined the effect of the water content on the depolymerization of the nanowires. Figure S8 shows the morphological evolution of the typical SEM images of the aggregates depolymerized from the nanowires at the various time at $40{ }^{\circ} \mathrm{C}$ (the morphology of the aggregates depolymerized from the nanowires shows no obvious change after $120 \mathrm{~h}$ ). The water content of nanowire solutions was $18.4 \mathrm{vol} \%, 23.8 \mathrm{vol} \%, 28.5 \mathrm{vol} \%$, and 32.6 vol\%, respectively. As shown in the Figures, the nanowires become more difficult to degrade into subunits as the water content is increased. When the water content increases to $23.8 \mathrm{vol} \%$, it was found that nanowire fragments and subunits were obtained. With increasing the water content to 32.6 vol $\%$, the nanowires were hardly depolymerized. The addition of the selective solvent (water) in the solution could enhance the inter-micelle interaction of the connected subunits, thus leading to difficulty in depolymerization. 
Water content

$5 \mathrm{~h}$

$12 \mathrm{~h}$

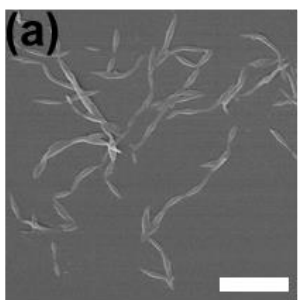

18.4 vol\%
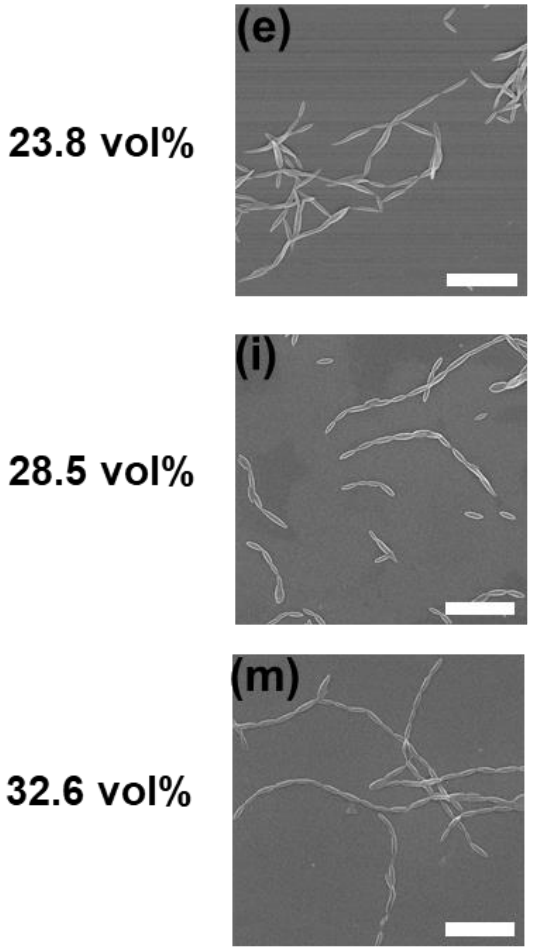
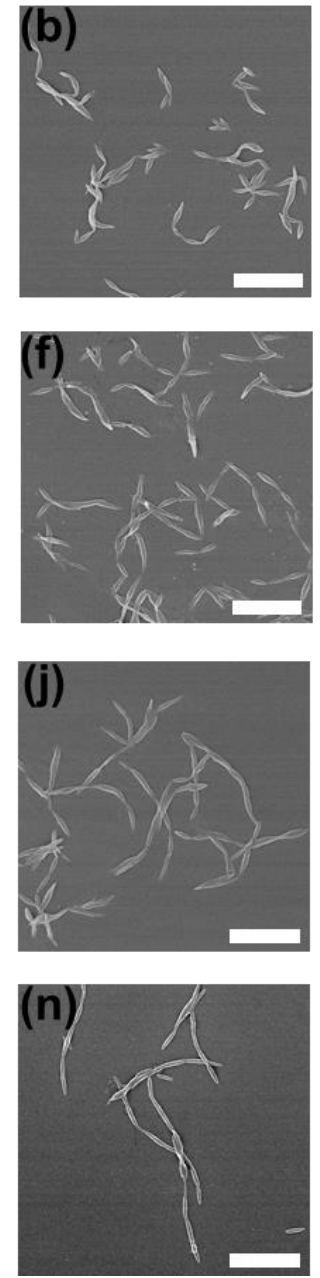

$48 \mathrm{~h}$

$120 \mathrm{~h}$
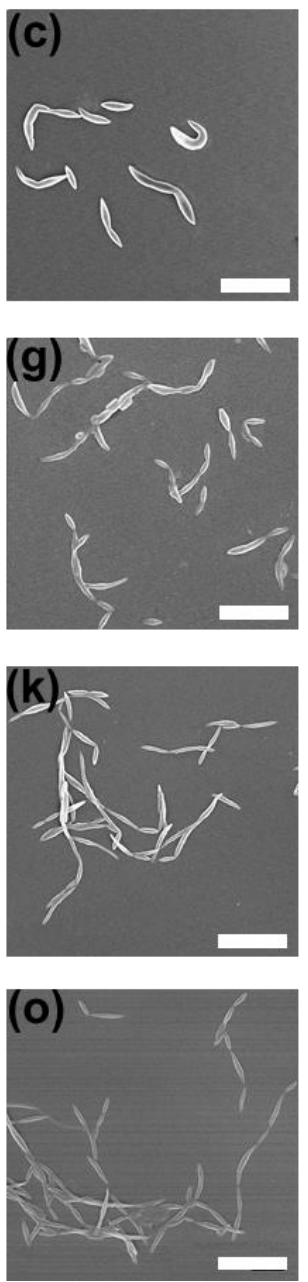
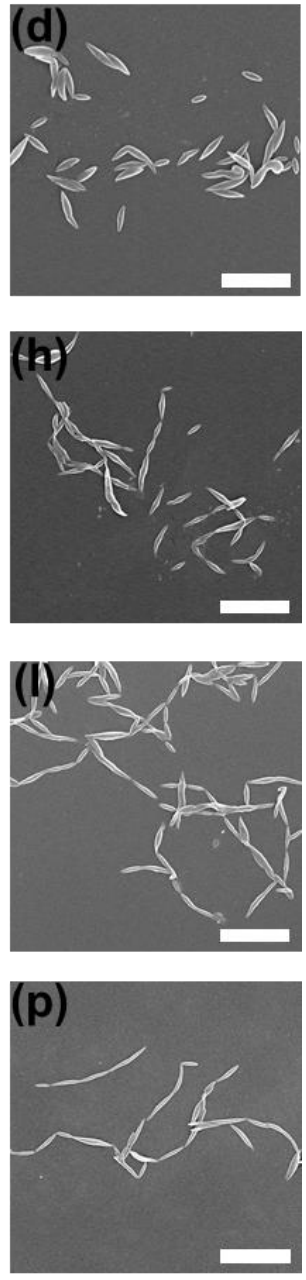

Figure S8. (a-p) SEM images of the aggregates depolymerized from the nanowires at $40{ }^{\circ} \mathrm{C}$ after depolymerization for 5, 12, 48, 120 hours. The water content is (a-d) $18.4 \mathrm{vol} \%$, (e-h) $23.8 \mathrm{vol} \%$, (i-l) 28.5 vol $\%$ and (m-p) 32.6 vol\%, respectively. Scale bars: $2 \mu \mathrm{m}$. 


\subsection{Effect of the inter-micelle interaction on the subunit volumetric changes}

We also examined the volumetric variation of the subunits of the nanowires by collecting SEM images with more than 400 micelles and analyzing the images using the Image-Pro Plus software. The statistical result of the subunit volume as a function of water content is shown in Figure S9. As can be seen, the average volume of the subunits decreases with the water content, which demonstrates that chain transfer between the subunits of the nanowires is prefer to occur at a lower water content.

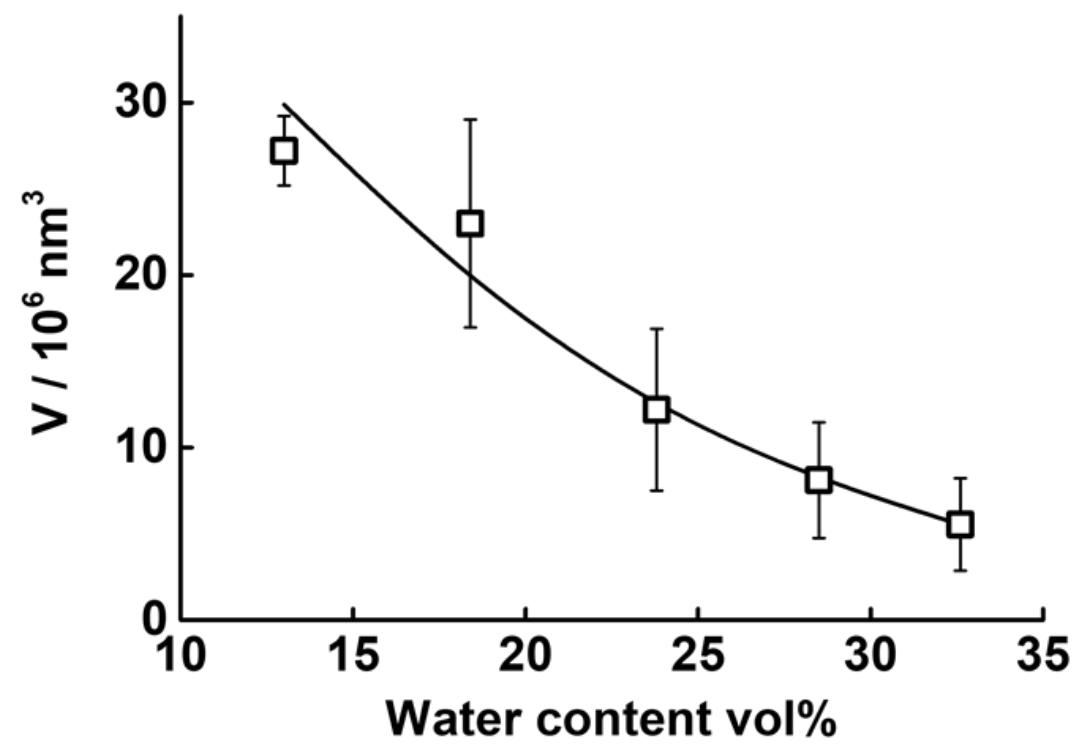

Figure S9. The volume of the subunits as a function of water content in solution. 


\section{References}

[S1] Blout, E.; Karlson, R. H., Polypeptides. III. The Synthesis of High Molecular Weight Poly- $\gamma$-Benzyl-L-Glutamates. J. Am. Chem. Soc. 1956, 78, 941-946.

[S2] Deming, T. J., Polypeptide and Polypeptide Hybrid Copolymersynthesis via NCA Polymerization. Adv. Polym. Sci. 2006, 202, 1-18.

[S3] Cai, C.; Lin, J.; Chen, T.; Tian, X., Aggregation Behavior of Graft Copolymer with Rigid Backbone. Langmuir 2010, 26, 2791-2797.

[S4] Zhuang, Z.; Jiang, T.; Lin, J.; Gao, L.; Yang, C.; Wang, L.; Cai, C., Hierarchical Nanowires Synthesized by Supramolecular Stepwise Polymerization. Angew. Chem. Int. Ed. 2016, 55, $12522-12527$.

[S5] Yang, C.; Ma, X.; Lin, J.; Wang, L.; Lu, Y.; Zhang, L.; Cai, C.; Gao, L., Supramolecular “Step Polymerization" of Preassembled Micelles: A Study of "Polymerization” Kinetics. Macromol. Rapid Commun. 2018, 39, 1700701.

[S6] Inomata, K.; Shimizu, H.; Nose, T., Phase Equilibrium Studies on Rod/Solvent and Rod/Coil/Solvent Systems Containing $\operatorname{Poly}(\alpha$, L-Glutamate) Having Oligo(ethylene glycol) Side Chains. J. Polym. Sci., Part B: Polym. Phys. 2000, 38, 1331-1340.

[S7] Jada, A.; Hurtrez, G.; Siffert, B.; Riess, G., Structure of Polystyrene-block-poly(ethyleneoxide) Diblock Copolymer Micelles in Water. Macromol. Chem. Phys. 1996, 197, 3697-3710.

[S8] Gan, Z.; Fung, J. T.; Jing, X.; Wu, C.; Kuliche, W. K., A Novel Laser Light-Scattering Study of Enzymatic Biodegradation of Poly(E-caprolactone) Nanoparticles. Polymer 1999, 40, 1961-1967.

[S9] Kratohvil, J. P., On Solution Properties of Poly( $\gamma$-benzyl-L-glutamate). Colloid Polym. Sci. 1970, $238,455-459$.

[S10] Higashihara, T.; Faust, R., Synthesis of Novel ABA Triblock and (ABA) $\mathrm{n}$ Multiblock 
Copolymers Comprised of Polyisobutylene and Poly( $\gamma$-benzyl-L-glutamate) Segments. React. Funct. Polym. 2009, 69, 429-434.

[S11] Kendrick, B. S.; Kerwin, B. A.; Chang, B. S.; Philo, a. J. S., Online Size-Exclusion High-Performance Liquid Chromatography Light Scattering and Differential Refractometry Methods to Determine Degree of Polymer Conjugation to Proteins and Protein-Protein or Protein-Ligand Association States. Anal. Biochem. 2001, 299, 136-146.

[S12] MacCallum, J. R., The Kinetics of Depolymerization of Addition Polymers. Eur. Polym. J. 1966, 2, 413-422.

[S13] Zeldin, M.; Jo, W. H.; Pearce, E. M., Kinetics of Thermal Degradation of Poly[bis(2,2,2-trifluoroethoxy)phosphazene]. Macromolecules 1980, 13, 1163-1167.

[S14] Pielichowski, K.; Njuguna, J., Thermal degradation of pdolymeric materials. Rapra Technology Limited, Shawbury, Shropshire, UK: 2005.

[S15] Ratnawati, R.; Prasetyaningrum, A.; Wardhani, D. H., Kinetics and Thermodynamics of Ultrasound-Assisted Depolymerization of K-Carrageenan. Bull. Chem. React. Eng. Catal. 2016, $11,48-58$.

[S16] Gao, H.; Ma, X.; Lin, J.; Wang, L.; Cai, C.; Zhang, L.; Tian, X., Synthesis of Nanowires via Temperature-Induced Supramolecular Step-Growth Polymerization. Macromolecules 2019, $52,7731-7739$.

[S17] Guerin, G.; Qi, F.; Cambridge, G.; Manners, I.; Winnik, M. A., Evaluation of the Cross Section of Elongated Micelles by Static and Dynamic Light Scattering. J. Phys. Chem. B 2012, $116,4328-4337$.

[S18] Liu, G.; Yan, X.; Duncan, S., Polystyrene-block-polyisoprene Nanofiber Fractions. 1. Preparation and Static Light-Scattering Study. Macromolecules 2002, 35, 9788-9793.

[S19] Fu, J.; Wu, C., Laser Light Scattering Study of the Degradation of Poly(sebacic anhydride) 
Nanoparticles. J. Polym. Sci., Part B: Polym. Phys. 2001, 39, 703-708.

[S20]Pedersen, J. S., Analysis of Small-Angle Scattering Data from Colloids and Polymer Solutions: Modeling and Least-Squares Fitting. Adv. Colloid Interface Sci. 1997, 70, 171-210.

[S21] Roig-Solvas, B.; Brooks, D.; Makowski, L., A Direct Approach to Estimate the Anisotropy of Protein Structures from Small-Angle X-Ray Scattering. J. Appl. Cryst. 2019, 52, 274-283.

[S22] Papadopoulos, P.; Floudas, G.; Schnell, I.; Lieberwirth, I.; Nguyen, T. Q.; Klok, H. A., Thermodynamic Confinement and $\alpha$-Helix Persistence Length in Poly( $\gamma$-benzyl-L-glutamate)- $b$-poly(dimethyl siloxane)- $b$-poly( $\gamma$-benzyl-L-glutamate) Triblock Copolymers. Biomacromolecules 2006, 7, 618-626.

[S23] Tang, H.; Ling, Y.; Deng, Y.; Zhang, D., Synthesis and Solid-State Self-Assembly of Poly(ethylene glycol)- $b$-poly( $\gamma$-benzyl-L-glutamate)s and Single-Walled Carbon Nanotubes. $J$. Polym. Sci., Part A: Polym. Chem. 2014, 52, 1905-1915. 